\title{
Endovascular repair using an AFX cuff for stent graft induced new entry involved visceral artery
}

\author{
Takayuki Kadohama ${ }^{1}$, kentaro kiryu ${ }^{2}$, Daichi Takagi ${ }^{1}$, Genbu Yamaura ${ }^{3}$, Fuminobu \\ Tanaka $^{1,3}$, Yoshinori Itagaki ${ }^{2}$, Takeshi Arai ${ }^{1}$, Takuya Wada ${ }^{3}$, Itaru Igarashi ${ }^{3}$, and Hiroshi \\ Yamamoto $^{4}$ \\ ${ }^{1}$ Akita University \\ ${ }^{2}$ Akita University Graduate School of Medicine School of Medicine \\ ${ }^{3}$ Akita University Graduate School of Medicine School of Medicine Department of \\ Cardiovascular Surgery \\ ${ }^{4}$ Akita University Graduate School of Medicine
}

June 30, 2020

\begin{abstract}
We encountered a challenging case of endovascular repair for distal stent graft-induced new entry (SINE) using the AFX aortic cuff. Postoperative computed tomography revealed no endoleak with a preferable conformation change of the externally mounted graft material of the AFX cuff against the tear.
\end{abstract}

\section{Introduction}

In the last decade, thoracic endovascular aortic repair (TEVAR) has emerged as an alternative to traditional aortic repair ${ }^{1}$. However, secondary interventions are relatively common in patients undergoing TEVAR procedures. The formation of a new tear at the margin of the stent graft is a major complication ${ }^{2-5}$. Distal stent graft-induced new entry (SINE) is that located within a few centimeters from the distal end of the stent graft, and the rate of observation is on the rise. This can be a lethal complication, and the rate of recurrence for distal SINE remains high in patients managed with secondary TEVAR ${ }^{2}$.

The AFX endograft system (Endologix, Irvine, CA, USA) uses novel high-density expanded polytetrafluoroethylene (ePTFE) graft material. The concept of active-seal fixation, which is unique to this device, stems from the loose conjunction of the fabric to the endoskeleton, thus allowing the conformable material to move independent of the stent and accommodate varied shapes ${ }^{6,7}$. To our knowledge, this is the first report in the literature of an AFX aortic cuff being used to treat distal SINE after primary TEVAR. The patient consented to this presentation.

\section{Case Report}

A 68-year old man with a medical history of Stanford type B acute aortic dissection 7 years ago was transferred to our institution for the treatment of aortic expansion 2 years before the present operation. We implanted a stent graft from immediately distal to the left common carotid artery (zone 2) to above the celiac artery using 2 pieces of Relay Plus (Terumo Corporation, Tokyo, Japan) endografts (proximal: 38 -34 tapered $\times 200 \mathrm{~mm}$, distal: $26 \times 150 \mathrm{~mm}$ ) concomitant with left carotid-subclavian artery bypass. The patient was discharged without complications. During the follow-up period, distal SINE involving expansion of the thoracoabdominal aorta occurred two years after primary TEVAR. The tear was located just at the 
distal end of the stent graft and it was located less than $20 \mathrm{~mm}$ from the origin of the super mesenteric artery (SMA) on computed tomography (CT) (Fig. 1).

The operation was carried out under general anesthesia in an operating room. The left femoral artery was exposed surgically for delivery of the endograft. Intravascular ultrasound imaging (Phillips Volcano, San Diego, CA, USA) was applied to ensure that a 0.035 Lundequist extra-stiff wire (COOK Medical, Bloomington, IN, USA) was introduced up to the level of the ascending aorta through the true lumen of the dissecting aorta. Moreover, a 4 French sheath was inserted into the contralateral common artery by a percutaneous puncture and an intraoperative angiogram was thus performed.

Ahead of the implantation of the endograft, a 0.035 Radifocus wire (Terumo Corporation, Tokyo, Japan) was cannulated into the SMA to visualize the origin of the artery on X-ray. After performing a preprocedural angiogram, an AFX aortic cuff measuring $28 \times 95 \mathrm{~mm}$ was deployed from the previous endograft to a site just above the origin of the SMA. Consequently, the celiac artery was covered with the endograft. The angiogram findings revealed no endoleakage and the presence of a satisfactory blood flow in the celiac artery via the SMA.

The postoperative course was uneventful without any abdominal symptoms. Follow-up CT revealed complete exclusion of the tear (Fig. 2). At approximately one year after surgery, he is recovering well without any symptoms.

\section{Discussion}

TEVAR has revolutionized the clinical approach to thoracic aortic pathology in the last decade. However, there are certain complications associated with this procedure, such as endoleaks, spinal cord ischemia and access-related injury, although the procedure is associated with a lower mortality and morbidity than open surgery $\left({ }^{4}\right)$. In addition, distal SINE is another life-threatening complication that can possibly result in aortic rupture. The incidence has been reported to range from $1.3 \%$ to $34.8 \%{ }^{2,5,8}$.

While the management strategy for distal SINE has included medical treatment, secondary TEVAR or open surgery, the indication remains controversial. Li et al. found that the recurrent rate of distal SINE was still high in patients managed with secondary TEVAR, possibly due to the fibrotic and less-mobile intimal flap in the chronic phase and use of less-tapered stent grafts ${ }^{2}$. To resolve this issue, an endograft with high adaptability as well as an adequate graft diameter is required. However, the majority of endografts are constructed by self-expanding stents sewn to the outer part of the fabric, and they have a certain radial expansion force which might cause a recurrence of SINE. The AFX endograft, by contrast, is unique in that the graft material is externally mounted and attached only at the proximal and distal ends of the stent, allowing it to move independent of the stent with a weakened radial expansion force. Therefore, it can accommodate various shapes, known as an "active-seal fixation", and it can effectively seal any tears (Fig. 3) ${ }^{6,7}$.

This approach makes it possible to effectively treat distal SINE, even though it is difficult to secure an adequate landing zone due to the presence of visceral arteries, as was seen in this case (Fig. 1). To our knowledge, this is the first report in the literature in which the AFX aortic cuff was used to treat distal SINE after primary TEVAR for chronic aortic dissection.

In conclusion, we encountered a challenging case of distal SINE after primary TEVAR in a case of chronic aortic dissection treated via an endovascular approach using the AFX aortic cuff. This tactic resulted in the successful treatment of the distal SINE. This may be a useful alternative option for treating focal thoracoabdominal aortic pathologies, such as SINE or saccular aneurysms, although a long-term follow-up is required.

\section{References}

1. Nienaber CA, Kische S, Rousseau H, et al. Endovascular repair of type B aortic dissection: long-term results of the randomized investigation of stent grafts in aortic dissection trial. Circ Cardiovasc Interv. 
2013;6(4):407-416.

2. Li Q, Ma WG, Zheng J, et al. Distal Stent Graft-Induced New Entry After TEVAR of Type B Aortic Dissection: Experience in 15 Years.Ann Thorac Surg. 2019;107(3):718-724.

3. Jang H, Kim MD, Kim GM, et al. Risk factors for stent graft-induced new entry after thoracic endovascular aortic repair for Stanford type B aortic dissection. J Vasc Surg. 2017;65(3):676-685.

4. Janosi RA, Tsagakis K, Bettin M, et al. Thoracic aortic aneurysm expansion due to late distal stent graft-induced new entry. Catheter Cardiovasc Interv. 2015;85(2):E43-53.

5. Weng SH, Weng CF, Chen WY, et al. Reintervention for distal stent graft-induced new entry after endovascular repair with a stainless steel-based device in aortic dissection. J Vasc Surg.2013;57(1):64-71.

6. Melas N, Stavridis K, Saratzis A, Lazarides J, Gitas C, Saratzis N. Active proximal sealing in the endovascular repair of abdominal aortic aneurysms: early results with a new stent-graft. $J$ Endovasc Ther.2015;22(2):174-178.

7. Diethrich EB. Novel sealing concept in the Endologix AFX unibody stent-graft. J Cardiovasc Surg (Torino). 2014;55(1):93-102.

8. Pantaleo A, Jafrancesco G, Buia F, et al. Distal Stent Graft-Induced New Entry: An Emerging Complication of Endovascular Treatment in Aortic Dissection. Ann Thorac Surg. 2016;102(2):527-532.

Figure Legend

Figure 1. Preoperative CT scan (A: Sagittal, B: axial, C: coronal). Note that there is $<20 \mathrm{~mm}$ from the tear (asterisk) to the origin of the super mesenteric artery (SMA: S).

Figure 2. Postoperative CT scan (A: Sagittal, B: axial, C: coronal). No endoleak and patent SMA (S). Fabric of the endograft has moved away from the stent to provide active-seal (arrows).

Figure 3. A postoperative 3D-CT angiogram. Satisfactory imaging of the celiac artery via the communicating SMA (arrow) and the tear has been covered with fabric of the endograft by active-seal fixation (asterisk). 


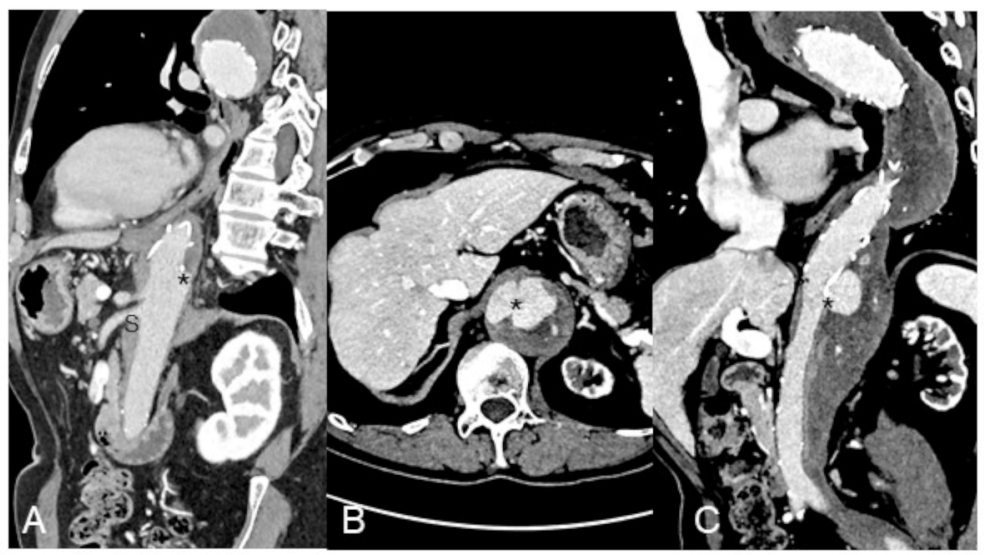

Figure 1 


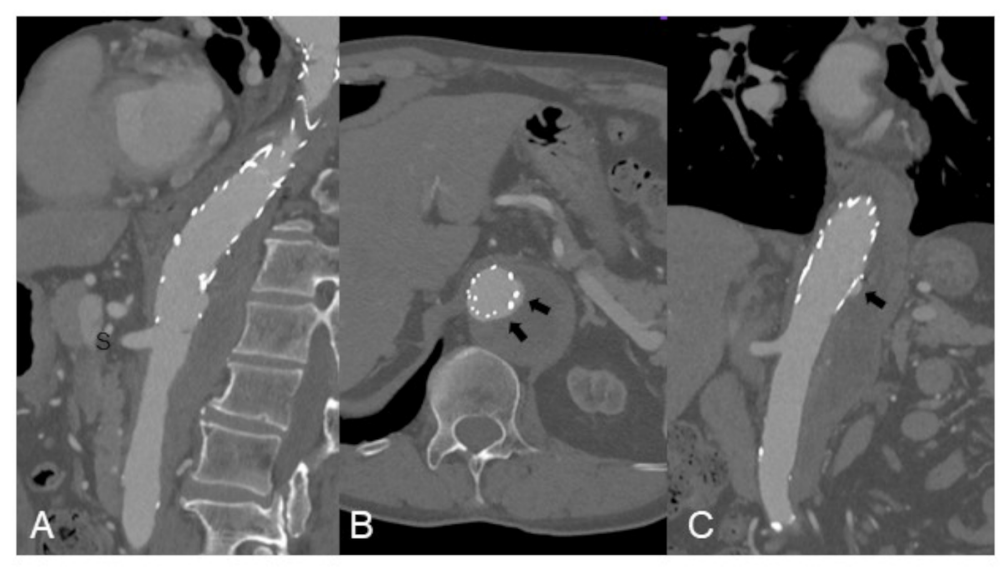

Figure 2 


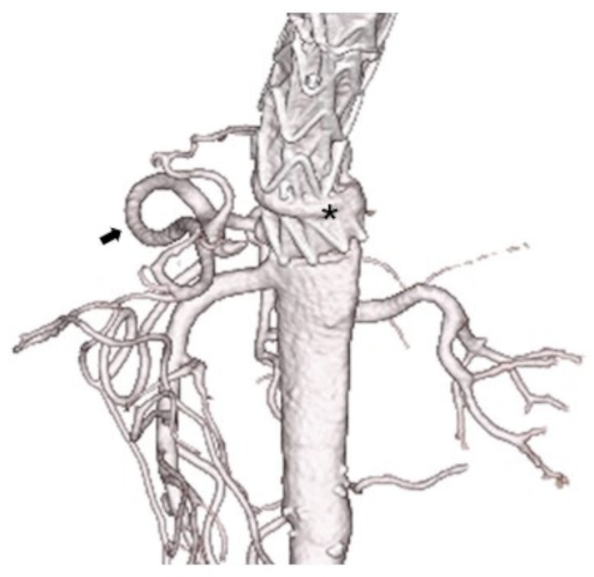

Figure 3 